\title{
Reativação da infecção por Trypanosoma cruzi em paciente com síndrome de imunodeficiência adquirida
}

\author{
Reactivation of Trypanosoma cruzi infection in a patient \\ with acquired immunodeficiency syndrome
}

\author{
Maria Clara G utierrez Galhardo, Ivana A. Martins, Alejandro Hasslocher- Moreno, \\ Sérgio Salles Xavier, Janice Mery Chicarino Coelho, Angela Cristina \\ Veríssimo Vasconcelos e Ricardo dos Santos Ribeiro
}

\begin{abstract}
Resumo Uma paciente com síndrome de imunodeficiência adquirida (SIDA) e doença de Chagas, com xenodiagnóstico positivo, estava em uso prolongado de cetoconazol com o objetivo de suprimir a parasitemia e prevenir a reativação da doença de Chagas. O cetoconazol foi suspenso inadvertidamente após 6 meses de uso. Um mês após, a paciente foi internada com febre, cefaléia, vômitos, taquicardia e hepatoesplenomegalia. Tanto o xenodiagnóstico como o exame de sangue a fresco demonstraram a presença de Trypanosoma cruzi. $O$ tratamento com benzonidazol foi instituído, com supressão da parasitemia. A paciente desenvolveu concomitantemente uma provável neurotoxoplasmose, evoluindo para o óbito em septicemia. À necropsia, não foram encontrados parasitas.
\end{abstract}

Palavras-chaves: HIV. SIDA. Doença de Chagas. Trypanosoma cruzi. Miocardite. Meningoencefalite.

\begin{abstract}
A patient with AIDS and asymptomatic Chagas's disease and positive xenodiagnosis was taking ketoconazole in order to supress parasitemia and prevent reactivation of Chagas's disease. Ketoconazole was unplanned suspended after 6 months, and the patient was admitted with fever, headache, vomiting, tachycardia, postural hypotension, hepatosplenomegaly, and positive xenodiagnosis one month later. Treatment with benzonidazole was begun leading to supression of parasitemia. The patient had probabily a neurotoxoplasmosis associated and progressed to coma and death with sepsis. No parasite was found in autopsy.
\end{abstract}

Key-words: HIV. AIDS. Chagas' disease. Trypanosoma cruzi. Myocarditis. Meningoencephalitis.

A reativação da doença de Chagas tem sido relacionada a situações que induzem imunodepressão, como neoplasias, drogas e, atualmente, a infecção pelo HIV1 57914 .

Areativação da doença de Chagas representa em muitas vezes a primeira infecção oportunística nos pacientes com infecção pelo HIV e a meningoencefalite e/ou miocardite são as principais manifestações clínicas2 3511 12. Embora o diagnóstico seja estabelecido e o tratamento específico instituído, a progressão é muitas vezes fatal4 15 .

O caso clínico descrito a seguir se refere a paciente com SIDA e reativação da doença de Chagas.

Laboratório de Pesquisa Clínica e de Anatomia Patológica do Centro de Pesquisa do Hospital Evandro Chagas e Departamento de Medicina Tropical, Laboratório de Imunopatologia do Instituto Oswaldo Cruz, Fundação Oswaldo Cruz, Rio de Janeiro, RJ, Brasil

Endereço para correspondência: Dra Maria Clara Gutierrez Galhardo. Centro de Pesquisa do Hospital Evandro Chagas/FIOCRUZ. Av. Brasil 4365, 21045-900 Rio de Janeiro, RJ, Brasil. Fax 5521 590-9988.

Recebido para publicação em 10/7/98. 


\section{RELATO DO CASO}

I.L., feminina, 45 anos, natural da Bahia, com diagnóstico de doença de Chagas na forma indeterminada, encontrava-se em controle ambulatorial desde 1987, com sorologia positiva $(\mathrm{IFI}=1 / 160, \mathrm{HA}=1 / 1280$ e ELISA $=1 / 1280)$ e xenodiagnósticos positivos em outubro de 1987, janeiro e dezembro de 1989 (5\%, 2,5\% e 27,5\% de ninfas positivas respectivamente). Em abril de 1990, foi realizada biópsia de gânglio cervical, que se encontrava-se aumentado, cujo exame microscópico sugeria infecção pelo HIV confirmada por sorologia (2 ELISAS e 1 IFI). Não sofrera hemotransfusões, negava uso de drogas injetáveis e o parceiro de então não era portador do HIV. Como neste ano a paciente apresentou uma infeção oportunística definidora de SIDA (diarréia por Isospora belli) optou-se pelo uso do cetoconazol na dose de $400 \mathrm{mg} / \mathrm{dia}$ por tempo prolongado com o objetivo de suprimir a parasitemia por Trypanosoma cruzi ( T. cruzi) e evitar a reativação da infecção. Após 70 dias de tratamento, o xenodiagnóstico de controle foi negativo. Apaciente interrompeu por conta própria o cetoconazol após 6 meses; um mês após a suspensão, foi internada com febre, cefaléia, vômitos, taquicardia, hipotensão postural, hepatoesplenomegalia e rigidez de nuca. $O$ exame do líquor revelou antígeno de $T$. cruzi utilizando IgG policlonal anti- T. cruzi (técnica de DOT-ELISA), apesar de o exame a fresco do líquor ter sido negativo. A pesquisa de tripomastigotas em gota fresca, gota espessa corada de sangue periférico e por xenodiagnóstico foi positiva ( $40 \%$ de ninfas positivas). Os eletrocardiogramas seriados e o ecocardiograma foram compatíveis com miocardite. Caracterizado o quadro clínico como reativação da doença de Chagas, iniciou-se benzonidazol (200mg/dia). No quarto dia de tratamento, não foram encontrados mais parasitas (gota fresca espessa e xenodiagnóstico). Apesar da negativação da parasitemia, a paciente apresentou deterioração do quadro neurológico, com sinais de localização. Atomografia computadorizada de crânio revelou lesão hipodensa, sem captar contraste, no tálamo à direita. Como a paciente havia apresentado melhora do quadro da doença de Chagas, optamos pelo tratamento empírico para neurotoxoplasmose devido a sorologia positiva para toxoplasmose (IFI 1/1024) a localização em tálamo e por ser a neurotoxoplasmose a principal causa de massa intracraniana em pacientes com SIDA.

O benzonidazol foi mantido por 45 dias, sendo substituído pelo cetoconazol $400 \mathrm{mg} / \mathrm{dia}$ como tratamento supressivo. Após a melhora clínica inicial, houve nova deterioração do quadro neurológico, e a paciente evoluiu para o óbito.

A necrópsia mostrou coração macroscopicamente normal, com miocardite e pericardite crônicas e a microscopia, não havia parasitas. Foram realizadas reações de imunohistoquímica pelo método da peroxidase (avidina-biotina) utilizando-se anticorpos policlonais anti-T. cruzi (cepa Y processados pelo IOC/FIOCRUZ) e anti-Toxoplasma gondii (ABC vector), que foram negativas.

O exame do encéfalo revelou leptomeninges delgadas e transparentes, pouco congestas. Aos cortes frontais sucessivos, os ventrículos tinham a capacidade habitual e notavamse áreas arredondadas ou irregularmente necróticas e/ou císticas à direita e nos gânglios basais bilateralmente; tais áreas exibiam histologicamente, necrose com perda de substância e formação de cavidades contendo macrófagos de citoplasma abundante, e reação astrocitária na periferia. Lesões de padrão histológico similar foram vistas em córtex cerebral, substância branca dos hemisférios cerebrais e cerebelares na ganglia basal, tálamo, cápsula interna, mesencéfalo, ao nível do lemnisco médio, e tegmento da ponte. Também não foram encontrados parasitos. Não foram realizados por problemas técnicos cortes em tubo digestivo. Havia adrenalite por citomegalovírus e broncopneumonia.

\section{DISCUSSÃO}

Embora seja controverso o uso de tripanossomicidas na fase crônica da doença de Chagas, a gravidade e o prognóstico reservado dos casos descritos de reativação da doença de Chagas em imunossuprimidos, motivaram-nos o tratamento inicial com cetoconazol nesta paciente que apresentava xenodiagnóstico positivo na vigência de imunodepressão associada à infecção pelo HIV. Tal opção de tratamento foi baseada na sensibilidade de algumas cepas de T. cruzi ao cetoconazol e no fato de esta ser uma droga menos tóxica que as demais drogas tripanossomicidas disponíveis8. Pareceu-nos que o cetoconazol foi capaz de 
controlar a parasitemia, pois todos os xenodiagnósticos anteriores ao uso de drogas tripanossomicidas eram positivos e, coincidentemente, um mês após a suspensão do medicamento, a paciente apresentou miocardite e comprometimento do SNC com intensa parasitemia, tal como é observado nos casos de reativação da doença de Chagas4 910121518 .

O tratamento com benzonidazol resultou em negativação da parasitemia, sem melhora clínica devido a uma possível associação com neurotoxoplasmose.

À necropsia, de modo semelhante a outros casos tratados de reativação da doença de Chagas associado a SIDA, não foram encontrados parasitas e os achados microscópicos não permitiram distinguir toxoplasmose, doença de Chagas (ou do próprio HIV, no caso da miocardite)4 1013 .
Acreditamos que, nesta paciente, o HIV modificou o curso da doença de Chagas, assim como a infecção crônica pelo $T$. cruzi agravou a imunodepressão pelo HIV, fato este já observado experimentalmente 17 . Portanto, é importante ficar atento aos casos de reativação da doença de Chagas em nosso meio, devendo o T. cruzi ser considerado um patógeno oportunístico, e nos casos de co-infecção pelo HIV, a reativação da doença de Chagas ser critério de SIDA2 12. Finalmente, alertamos que, com a expansão da infecção pelo HIV na população residente nas grandes cidades e a urbanização da doença de Chagas, resultante da migração dos indivíduos infectados da região rural, novos casos de infecção concomitante poderão surgir. Isto poderá ser agravado pela transmissão de ambos os patógenos por transfusão sangüínea e/ou hemoderivados, conseqüência do erro das sorologias empregadas ou mesmo falta de um rigoroso controle dos doadores de sangue ${ }^{16}$.

\section{REFERÊNCIAS BIBLIOGRÁFICAS}

1. Almeida EA, Engelman DES, Metze K, Teixeira MAB, Tostes Jr S, Lopes ER. Encefalopatia chagásica em imunodeprimido por tratamento quimioterápico em portador de leucose crônica: apresentação de caso com necrópsia. Revista da Sociedade Brasileira de Medicina Tropical 24(Supl.I):33, 1991.

2. Del Castillo M, Mendoza G, Oviedo J, Bianco RPP, Anselmo AE, Silva M. AIDS and Chagas'disease with central nervous system tumor-like lesion. The American Journal of Medicine 88:693-694, 1990.

3. Ferreira MS, Nishioka SA, Rocha A, Silva AM, Ferreira RG, Oliver W, Tostes Jr S. Acute fatal Trypanosoma cruzi meningencephalitis in a human immunodeficiency viruspositive hemophiliac patient. The American Journal of Tropical Medicine and Hygiene 45:723-727, 1991.

4. Ferreira MS, Nishioka SA, Silvestre MTA, Borges AS, Nunes-Araújo FRF, Rocha A. Reactivation of Chagas' disease in patients with AIDS: report of three new cases and review of the literature. Clinical Infectious Diseases 25:1397-1400, 1997.

5. Gallo P, Fabião Neto OM, Suarez JMM, Borba RP.Acute central nervous system infection by Trypanosoma cruzi and AIDS. Arquivos de Neuro-Psiquiatria 50:375-377, 1992.

6. Gluckstein D, Ciferri F, Ruskin J. Chagas' disease: another cause of cerebral mass in the acquired immunodeficiency syndrome. The American Journal of Medicine 92:429-432, 1992.

7. Kirchhoff LV. American trypanosomiasis (Chagas' disease) - a tropical disease now in the United States. New England of Medicine 329:639-644, 1993.
8. McCabe RE, Remington JS, Araújo FG. Ketoconazole inhibition of intracellular multiplication of Trypanosoma cruzi and protection of mice against lethal infection with the organism. The Journal of Infectious Diseases 150:594-601, 1984.

9. Metze K, Maciel Jr JA. AIDS and Chagas' disease. Neurology 43:447-448, 1993.

10. Nishioka SA, Ferreira MS, Rocha A, Burgarelli MKN, Silva AM, Duarte MIS, Schmitt FC. Reactivation of Chagas' disease successfully treated with benznidazole in a patient with acquired immunodeficiency syndrome. Memórias do Instituto Oswaldo Cruz 88: 493-496, 1993.

11. Oddó D, Casanova M, Acuña G, Ballesteros J, Morales B. Acute Chagas' disease (Trypanosomiasis americana) in acquired immunodeficiency syndrome: report of two cases. Human Pathology 23: 41-44, 1992.

12. Rocha A, Ferreira MS, Nishioka SA, Burgarelli MKN, Silva AM, Moura LP, Ugrinovich R, Raffin CN. Trypanosoma cruzi meningoenphalitis and myocarditis in a patient with acquired immunodeficiency syndrome. Revista do Instituto de Medicina Tropical de São Paulo 35:205-208, 1993.

13. Rocha A, Meneses ACO, Silva AM, Ferreira MS, Nishioka SA, Burgarelli MKN, Almeida E, Turcato Jr G, Metze K, Lopes ER. Pathology of patients with Chagas' disease and acquired immunodeficiency syndrome.The American Journal of Tropical Medicine and Hygiene 50:261-268, 1994.

14. Rosemberg S, Chaves CJ, Higuchi ML, Lopes MBS, Castro LHM, Machado LR. Fatal meningoencephalitis caused by reactivation of Trypanosoma cruzi infection in a patient with AIDS. Neurology 42:640-642, 1992. 
15. Sartori AMC, Shikanai-Yasuda MA, Amato Neto V, Lopes $\mathrm{MH}$. Follow-up of 18 patients with human immunodeficiency virus infection and chronic Chagas' disease, with reactivation of Chagas' disease causing cardiac disease in three patients. Clinical Infectious Diseases 26:177179, 1998.

16. Schmuñis GA. Trypanosoma cruzi, the etiologic agent of Chagas's disease: status in blood supply in endemic and nonendemic countries. Transfusions 31:547-557, 1991.
17. Silva JS, Barral-Neto M, Reed SG. Agravation of both Trypanosoma cruzi and murine leukemia virus by concomitant infections. The American Journal of Tropical Medicine and Hygiene 49: 589-597, 1993.

18. Solari A, Saavedra $H$, Sepúlveda $C$, Oddó D, Acuña G, Labarca J, Muñoz S, Cuny G, Brengues C, Veas F, Bryan RT. Successful treatment of Trypanosoma cruzi encephalitis in a patient with hemophilia and AIDS. Clinical Infectious Diseases 16:255-259, 1993. 